\title{
Las escenificaciones como herramienta metodológica de la antropología*
}

\author{
Staging as a Methodological Tool \\ for Anthropology
}

\author{
Miren Urquijo ${ }^{1}$ \\ Universidad del País Vasco-Euskal Herriko Unibertsitatea
}

\section{RESUMEN}

Mi experiencia teatral condicionó mi acercamiento a la antropología. Habituada al enfoque dramatúrgico en el estudio de la vida social, esta circunstancia promovió la articulación de mi experiencia teatral y antropológica en las escenificaciones. Este artículo expone cómo y por qué esta herramienta es válida para la antropología y asimismo, para el estudio performativo del sistema de género y la apreciación sobre el papel de la normatividad y la estructura social en su representación. El proceso de construcción de una lógica dramatúrgica y antropológica para las escenificaciones lo he experimentado y expresado como un ejercicio autobiográfico. Las teorías sociales que me han afectado han sido el situacionismo dinámico, el interaccionismo, y las teorías constructivistas que tratan al cuerpo como ficción social. Con todo ello he elaborado una estrategia interpretativa para la descripción, percepción, y el análisis de la realidad. Publicarla es compartirla y someterla a evaluación.

Palabras clave: Género; Performatividad; Antropología.

\section{SUMMARY}

My theatrical background has conditioned my approach to anthropology. This dramaturgical focus in the study of social life has encouraged the articulation of my theatrical and anthropological experience in staging. This paper explains how and why this is a valid tool for anthropology and for the performative study of the gender system, the assessment of the role of normativity and social structure in its representation. I have experimented with and expressed the process of constructing a dramaturgical and anthropological logic as an autobiographical exercise. The social theories which have influenced me include dynamic situationism, dynamic interactionism and

${ }^{*}$ Una versión previa de este artículo fue publicada en euskera (Urquijo 2016), dando continuidad a un aspecto de la investigación promovida por mi tesis doctoral y su consecuente publicación (Urquijo 2011). Agradezco a Maggie Bullen, Mari Luz Esteban, Olatz González-Abrisketa, Jone Miren Hernández, Lidia Montesinos y María Zapata la amable revisión de los manuscritos originales y sus atentos comentarios. Agradezco también a los o las revisoras sus acertados comentarios, que me han obligado a reconstruir el artículo, mejorándolo. Agradezco por último la ayuda y financiación recibida en el Grupo de Investigación del Sistema Universitario Vasco: A.F.I.T. Antropología Feminista Ikerketa Taldea/Grupo de Investigación en Antropología Feminista; Referencia: IT1030-16.

${ }^{1}$ Correo electrónico: miren.urquijo@ehu.eus. ORCID iD: http://orcid.org/0000-0002-5237-5352. 
constructivist theories which address the body as social fiction. With all of this, I have elaborated an interpretative strategy for the description, analysis and perception of reality. Publishing it means sharing it and submitting it to evaluation.

Key words: Gender; Performativity; Anthropology.

El feminismo ama otra ciencia: las ciencias y las políticas de la interpretación, de la traducción, del tartamudeo y de lo parcialmente comprendido (Haraway 1995: 336).

La teoría feminista ha cuestionado críticamente la autoridad y objetividad del positivismo científico en las ciencias sociales, reivindicando el papel del subjetivismo y del yo reflexivo en la práctica académica e investigadora. En lugar del objetivismo transcendental con su mirada "desde ningún lado", la versión feminista de la objetividad requiere ubicaciones limitadas y conocimientos situados (Haraway 1995). El ejercicio autobiográfico ligado a la memoria resulta por tanto un género relevante para la construcción del conocimiento, donde el pasado de la persona investigadora adquiere importancia para comprender su elección de las áreas de estudio, su experiencia y análisis del trabajo de campo, y su escritura (Valle 1995; Hernández 1999). Teresa del Valle (1995) en sus estudios sobre la memoria ha detectado una serie de claves metodológicas para la elaboración de las autobiografías, proporcionando un conjunto de pautas para el proceso de selección de recuerdos, que tienen mucho de recorte consciente y selectivo. Así, en lo que sigue construiré, a partir de las claves propuestas por esta autora, un ejercicio en torno a: los hitos (referencias significativas, vivencias y decisiones clave), las articulaciones (procesos de ajuste o enlace de las distintas partes de un todo) y los intersticios (momentos precursores de cambios) de mi autobiografía. Como mi intención en este artículo es reconocer cómo llego a la herramienta de las escenificaciones, construiré una particular versión del texto de Leszek Kolankiewics "Towards an Anhropology of Performance(s)" (2008) desde mi ubicación en aquellos hitos que han articulado intersticios autobiográficos desde lo teatral hacia lo antropológico.

Decidí dedicarme al teatro durante el montaje de la obra Woyzeck, de Georg Büchner ${ }^{2}$. Me subyugó la historia social del teatro reconstruida durante los ensayos. A continuación, actué en Yvonne, princesa de Borgoña, de Witold Gombrowicz ${ }^{3}$, interpretando las constricciones de la estructura social en la siempre inauténtica forma individual. Seguidamente cursé los estudios oficiales de Arte Dramático en Madrid, formándome en la escena naturalista de Constantin Stanislavski y Lee Strasberg ${ }^{4}$, aunque

${ }^{2}$ El texto teatral Woyzeck, de Büchner (1813-1837), es considerado precursor del expresionismo alemán. Basada en la historia real de su protagonista, representa una crítica sobre las condiciones sociales de explotación, abuso y humillación que sufre por parte de los estamentos superiores y le conducen hacia la tragedia.

${ }^{3}$ La obra de Witold Gombrowicz (1904-1969), autor vanguardista polaco, se caracteriza por una crítica corrosiva y tragicómica de las convenciones sociales impuestas sobre la libertad individual.

${ }^{4}$ George II, Duque de Saxe-Meiningen, perseguía con su compañía de teatro a finales del siglo XIX, "el arte como vida. Representar el movimiento, el inexorable progreso de la acción" (en Ceballos 1992: 12). Este director teatral produjo la revolución escénica naturalista en todos los 
algunos profesores defendieran la dialéctica de Bertolt Brecht. Finalmente, resultaron vivencias clave un seminario sobre la antropología teatral de Eugenio Barba ${ }^{5}$. Todo este bagaje agudizó mi percepción dramatúrgica de la vida social. En 1996 me matriculé en la Licenciatura de Antropología Social y Cultural. La mirada dramatúrgica comenzó a virar hacia la antropología. Y comencé entonces a percibir que la metáfora del mundo como teatro, repetida durante siglos en la literatura ${ }^{6}$, aparece a menudo en las humanidades y en las ciencias sociales a partir de la introducción en 1930 del concepto de rol social (Kolankiewicz 2008: 12) por representantes de varias disciplinas $^{7}$.

La antropología de la experiencia ${ }^{8}$, perspectiva formulada inicialmente por Turner (1986) inspirado por el pensador alemán Willhelm Dilthey (1833-1911) y su concepto de experiencia/vivencia, erlebnis, que expresa - lo vivido a través de-, resulta pertinente para explicar mi proceso. Según este enfoque, entendemos a otras personas y sus expresiones en base a nuestra propia experiencia y entendimiento, ya que la experiencia estructura nuestras expresiones. Creamos las unidades de experiencia y significado desde la continuidad de la vida. Cada historia tiene una imposición arbitraria de significado en el fluir de la memoria y, así, remarcamos ciertas causas y desechamos otras. Cada historia es interpretativa. Por tanto, como la experiencia está culturalmente construida mientras que el entendimiento presupone experiencia, esta estructuración entre experiencia y expresiones se produce en una espiral de evolución histórica, una construcción y reconstrucción progresiva (Turner y Bruner 1986: 3-6).

En mi historia, por una parte, la disección de la vida social para reconstruirla sobre el escenario en una escena naturalista (con sus protagonistas, antagonistas y conflictos, en unas determinadas circunstancias, activadoras interpretativas de la memoria emocional y la memorial sensorial) me hizo sensible al situacionismo de Max Gluckman, Clyde Mitchell y Victor Turner, al interaccionismo de Erving Goffman, al actor plural de Bernard Lahire o al actor-red de Bruno Latour. Por otra parte, la dramaturgia dis-

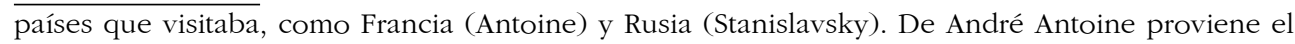
concepto de cuarta pared del teatro naturalista y del método interpretativo de Constantin Stanislavsky: el proscenio funciona como una cuarta pared del lugar en el que se desarrolla la acción dramática para que los actores puedan acentuar la ilusión de realidad sin tener en cuenta la presencia del público (Ceballos 1992: 42). A partir del sistema Stanislavski, Lee Strasberg (19011982) crea en el Actors Studio de Nueva York el conocido como el método, un conjunto de técnicas de actuación teatral con énfasis en la experimentación de la vida del personaje sin pensar dónde termina la acción personal y empieza la del personaje. Allí estudiaron, entre otros/as, Paul Newman, Al Pacino, Marlon Brando, Marilyn Monroe y Robert de Niro.

5 Eugenio Barba (1936), alumno de Grotowski, fundador del Odin Teatret en Oslo y del ISTA (The International Scholl of Theater Anthropology), centro para el intercambio transcultural y la investigación de la antropología teatral definida como estudio del ser humano en una situación de representación (Ceballos 1992: 306).

${ }^{6}$ En la literatura dramática, por ejemplo, son ampliamente conocidas la obra El gran teatro del mundo de Calderón de la Barca, o las palabras "All the world's a stage, / And all the men and women merely players" de la obra As you like it, de William Shakespeare

${ }^{7}$ Kolankiewicz cita a William James, Georg Simmel, Roberts E. Park, George Herbert Mead y otros (2008: 12-20).

8 "La antropología de la experiencia trata cómo los individuos experimentan su cultura realmente, esto es, cómo los acontecimientos son recibidos por la conciencia" (Turner y Bruner 1986: 4). La traducción es mía. 
tanciada y dialéctica de Bertolt Brecht y la antropología teatral de Eugenio Barba y Nicola Savarese me hizo reflexionar sobre la ficción social del cuerpo y allanó el camino hacia mi comprensión del trabajo de Pierre Bourdieu, Judith Butler y Mari Luz Esteban.

En definitiva, comencé a denominar escenificaciones al resultado de este cruce de perspectivas que me permite desarrollar una estrategia descriptiva, una estrategia analítica y una estrategia perceptiva en la investigación antropológica feminista.

\section{APUNTES PARA LA PRÁCTICA DE LAS ESCENIFICACIONES ETNOGRÁFICAS}

Observar una escenificación durante el trabajo de campo antropológico significaría adoptar una perspectiva dramatúrgica sobre la vida social, es decir, utilizarla como estrategia perceptiva. Además, las escenificaciones como herramientas analíticas pretenderían ser un procedimiento susceptible de validarse. Y como herramienta descriptiva, las escenificaciones serían representaciones de la vida social con las que pretendería describir, mediante una estrategia dramatúrgica, los datos etnográficos más relevantes y su análisis.

En su génesis he operado a partir de mi experiencia, componiéndolas sobre la base de elementos dispares, restos de otras creaciones reunidos y vueltos a armar mediante una forma de lógica combinatoria que Lévi-Strauss describió como del bricoleur. Ilustraré el proceso seguido con varios apuntes de escenificaciones tomados de una investigación que actualmente curso. Necesariamente, son bocetos esquemáticos cuyo único fin es el de ejemplarizar la propuesta.

La investigación se centra en diversas obras escénicas que han sido representadas el 2016 en un medio natural, objeto de estudio que por brevedad denomino naturaleza escénica. Partí de la hipótesis de que la naturaleza escénica es buena para pensar las relaciones de continuidad y discontinuidad entre naturaleza y cultura. Posteriormente esta hipótesis fue ampliada hacia las relaciones entre naturaleza, cultura y género. Y ahora estoy considerando la pertinencia de integrar el análisis cognitivo de la investigación en un análisis social, económico y político. Los casos que analizo son tres: a) El sueño de una noche de verano de Shakespeare, escenificado como itinerario en el parque Cristina Enea de Donostia durante las noches de julio de su capitalidad cultural; b) El espectáculo de danza Topaguneak, creado por el grupo Kukai en el museo Chillida Leku (Hernani, Gipuzkoa); c) El concierto Txoriei, por la orquesta de cuerda Et Incarnatus, al que asistí una tarde-noche de San Juan en un bosque de Tolosa (Gipuzkoa)?

Debo aclarar que aunque denomino naturaleza a los escenarios de estas obras, ninguna lo es en sentido estricto, aunque no sea este el lugar para abordar ese debate. El bosque del Sueño se representó en el parque Cristina Enea, diseñado por el jardinero Pierre Ducasse en terreno de dos caseríos que su dueño el Duque de Mandas había comprado en 1863 para construirse un palacio. Topaguneak se bailó en distintos puntos de las trece hectáreas de prado arbolado del museo Chillida-Leku,

\footnotetext{
9 Txoriei significa 'a los pájaros' y Topaguneak 'lugares de encuentro'. En adelante denominaré estas tres obras y sus naturalezas escénicas Txoriei, Topaguneak y Sueño.
} 
creado por Eduardo Chillida en el caserío Zabalaga para exponer sus esculturas. El concierto Txoriei, sobre los pájaros se celebró en un hayedo trasmocho, árboles cuya forma característica se logra mediante una poda destinada al mejor aprovechamiento humano de sus ramas. Es decir, en todos los casos la naturaleza ha sido un híbrido de naturaleza y cultura que interacciona con la cultura teatral, musical o danzante.

La experiencia/vivencia, erlebnis, que estructuró la percepción y construcción del objeto de estudio que investigo actualmente fue la asistencia a los espectáculos teatrales Xalbador y Blodeuwedd. En 1991 asistí en Larraine (Zuberoa, País Vasco francés) a la representación de la pastoral ${ }^{10}$ Xalbador. La acción dramática se vio enriquecida con el espectáculo ofrecido por las montañas pirenaicas ante las que estaba situado el tablado. La sombra de las nubes corriendo sobre los prados y las sucesivas tonalidades de verdes fueron más que un bello decorado para la obra sobre el poeta y pastor Xalbador. El año 2013, durante una estancia post-doctoral, fui invitada al espectáculo Blodeuwedd $^{11}$ en Tomen y Mur (Parque Nacional Snowdonia, Gales). En la entrada del parque nos recibió un mayordomo mientras unos soldados de la segunda guerra mundial nos observaban junto a un jeep. Desconcierto. Caminamos tras el mayordomo por un paisaje desolado hasta las cercanías de una granja en ruinas. Allí, en mitad de una colina, únicamente dos sofás chester o una mesita auxiliar con un juego de whisky en cristal tallado contextualizaban a los personajes en un salón burgués. La actuación era excelente. Sus gestos, entonación y vestuario te transportaban a otra época y lugar. Pero el contraste entre el refinamiento de la escena, la ruina de la granja, los árboles, el césped, las colinas y la identidad de los personajes —en un espacio temporal entre la Segunda Guerra Mundial y la leyenda celta-, unido al sofisticado sistema tecnológico de traducción simultánea del gaélico al inglés, provocaban una curiosa disonancia cognitiva que situaba lo percibido en un lugar intermedio que incluía las cualidades de cada parte. La representación de la obra continuó en otras localizaciones del parque. Nuestro caminar junto a una central nuclear, la transformación final de la protagonista en lechuza u otras escenificaciones de ese día merecerían ser analizadas más extensamente. Pero será abandonada aquí.

El objeto de mi estancia post-doctoral en la Universidad de Aberystwyth se situaba en el intersticio entre la performance, la antropología y el paisaje. Encontrar una formulación para las escenificaciones era uno de sus objetivos específicos. Habiendo advertido con anterioridad su potencial como herramientas descriptivas, perceptivas y analíticas, la estancia tuvo como resultado el no sentirme sola en el intento. Ahora, unos años después, recupero su impulso al compartir esta propuesta y debatirla. Presentaré en distintos apartados sus tres estrategias complementarias:

\footnotetext{
${ }^{10}$ La pastoral suletina es un género teatral de origen medieval, cuya dramaturgia gira en torno al dualismo entre el bien y el mal. Se representa por prácticamente todos los habitantes de una misma localidad y su escenario se sitúa al aire libre en un punto de ella. Cada año cambia su personaje protagonista y el municipio de acogida.

${ }^{11}$ Leyenda gaélica dramatizada en 1923-25 por el autor Saunders Lewis con el título añadido The Woman Made of Flowers.
} 


\section{LAS ESCENIFICACIONES COMO ESTRATEGIA DESCRIPTIVA}

Para Erika Firscher-Lichte, el concepto de escenificación implica un plan, elaborado por un autor/autora que "puede prever qué elementos serán empleados, en qué lugares, en qué momentos y de qué modo serán empleados" (Fischer-Lichte 2011: 104). Así, cada escenificación implica un plan, un plan dramatúrgico sobre esa descripción etnográfica, el cual no necesariamente se traduce en un texto con apariencia teatral. Puede quedar velado tras su estructura. Las escenificaciones, por otra parte, son figuras fractales, están compuestas de otras menores. Las escenificaciones —-secundariasde las escenificaciones —-superiores— son también escenificaciones en sí mismas, muy similares a las escenificaciones adyacentes que llevan una existencia independiente. La monografía como escenificación sería múltiple en la medida que es el precipitado de una multitud de relaciones genealógicas ${ }^{12}$ que se manifiestan de una en una. Al mismo tiempo, un conjunto de escenificaciones son una escenificación a causa de configurar una sola genealogía, como encadenamiento de escenificaciones, en las que unas se proyectan en las otras, brotan, en una relación integral.

\section{LAS ESCENIFICACIONES COMO ESTRATEGIA PERCEPTIVA}

Observar una escenificación durante el trabajo de campo antropológico significa adoptar una perspectiva que se refiere a observar la vida social como si fuera un teatro que girara en torno a la acción, la interacción y el conflicto como elementos básicos de la estructura del drama; esto es, observar el comportamiento objetivamente perceptible de los seres humanos, evaluar sus esquemas de acción incorporados durante las socializaciones, los diferentes mecanismos y lógicas de esa acción y seguir a un actor, femenino o masculino, mientras resuelve conflictos en esas situaciones e interacciones. En este sentido, es como si nos situáramos ante una obra de teatro naturalista en la que todos los elementos nos crearan la ilusión de participar de la acción escénica.

Sin embargo, como indica Roger D. Abrahams (1986), en el trabajo antropológico se produce una doble conciencia de la experiencia. Participamos en la acción, pero también informamos de ella. Somos parte y testigos de esa experiencia. La etnógrafa participa al mismo tiempo que observa, como en el trabajo actoral. La actuación y la etnografía serían ambas reflexivas en la atención dada al yo en la representación, y tanto en la investigación antropológica como en la interpretación actoral se está involucrado en la acción pero al propio tiempo fuera de ella para comunicarla efectivamente.

En este sentido, la reflexividad escénica durante el trabajo de campo contribuye a poder construir un distanciamiento, un extrañamiento. Más necesario, si cabe, cuando se trabaja en la propia cultura. Por ello mi conocer, mi tener experiencia previa sobre el extrañamiento brechtiano, ha sido una guía. El efecto de distanciamiento de Bertolt

\footnotetext{
12 "El concepto de genealogía (...) es el tropo crucial para hacer singular la pluralidad, y plural la singularidad" (Gell 2016: 185). Alfred Gell se remite a Marilyn Strathern y Roy Wagner en su análisis artístico de las figuras fractales. Yo les parafraseo.
} 
Brecht persigue un distanciamiento emocional con respecto a lo representado para evitar la inmersión del público en un mundo ilusorio y facilitar su reflexión crítica. Así, comencé a utilizar las escenificaciones como estrategias perceptivas para construir distancia y extrañamiento, como artefactos para problematizar lo cotidiano, lo cercano, aquello que se presentaba ante mí como lógico por conocido, como dispositivos para instalarme en la extrañeza, en la actitud de quien se asombra (Brecht 1983). Todo lo cual me condujo a una primera formulación de las mismas como artefactos objetivadores a través de la distancia (Urquijo 2011). La batería de unidades de análisis que guían el trabajo de campo proporciona un dispositivo clave para este distanciamiento.

Presentaré a continuación tres esbozos de escenificaciones en mi actual investigación. Todas responden a la misma unidad de análisis, que es el estudio de la agencia de la naturaleza escénica sobre el habitus de quienes participan en los espectáculos citados. Una unidad que premeditadamente invierte el estatus de control por parte de la cultura sobre la naturaleza, característico de nuestra europea concepción sobre la interacción naturaleza/cultura.

En el Sueño percibí escenificaciones en torno a puertas y escaleras. En la entrada al bosque, franqueada por dos grandes espejos de marco dorado junto al que cantaba elevado sobre una escalera un coro infantil, sentí la primera puerta al espacio de los sueños y recibí una bujía de luz para el resto del recorrido, nocturno. Más tarde, el ascenso por una escalera cavada en la tierra, en fila india junto a marcos de blancas puertas en los que bailaban los duendes y hadas (convertidos en blancos niños del sueño) por una parte incrementó mi sentido de realidad al tener que caminar en estado de alerta y reajuste corporal y por otra, me transportó hacia el espacio del sueño. Y durante la siguiente ascensión por el irregular terreno unos músicos de viento, dentro de marcos dorados suspendidos en el aire, tocaban melodías que luego punteaban distintos momentos de las escenas en las que los personajes fueron hechizados por la flor de Oberón. Las escenas de bosque de la obra interactuaban con el parque y esa naturaleza escénica desencadenaba un reajuste continuo de mis sentidos y activaba transformaciones cognitivas entre el sueño y la realidad.

Asimismo, tras Topaguneak escribí: "Nada más traspasar la puerta se te abre el alma. El día había sido lluvioso y brillaban las hojas de los árboles. Fui con katiuskas y pude caminar mucho fuera de los senderos. Cuidada hierba, olor a menta y tierra mojada. Las esculturas de Chillida y el público disperso a su alrededor, haciendo fotos. Los bailarines, vestidos de blanco, interactuaban casi inmóviles con algunas esculturas, y el paisaje. Llegué estresada y muy cansada, pero en pocos minutos sentí paz". Y en esa interacción pude apreciar la naturaleza escénica como agente de las transformaciones corporales de la audiencia y bailarines.

Finalmente, en Txoriei el hayedo, que constituía un anfiteatro natural, desencadenó una performance corporal en claro contraste con la habitual en los conciertos de cuerda en auditorios musicales: descalza, en ropa deportiva, tumbada a ratos, disfrutando al tiempo los rayos del sol colándose entre las hojas y la música, inmersa en una escenificación donde se hibridaba la naturaleza y la cultura acompañada de la hibridación entre cultura popular y alta cultura, del mismo modo que en el Sueño se combinaban ilusión y realidad. ¿Fue esa agencia la que provocó la exclamación de una compañera "Ha sido maravilloso, pero si esta música (contemporánea) la escuchas en casa igual apagas la radio"? ¿O fue algo más? 
En cualquier caso, la naturaleza escénica actuó sobre el habitus y performatividad del cuerpo de sus pacientes y su percepción de esas categorías, tanto sobre quienes fueron agentes de las obras escénicas como sobre quienes asistíamos a ellas. Y necesité de la hibridación entre empatía y distanciamiento para percibirla. No obstante, este bosquejo de escenificaciones es parcial y excesivamente personal. Falta incluir en el mismo los diálogos del público, las entrevistas a los directores de esas obras, a los/as actores, bailarines/as, músicos/as, a sus productoras, etc. En primer lugar, por ser deudoras del análisis situacionista; en segundo lugar, por ofrecer un truncado marco interactivo; y en tercer lugar, por su inconclusa incidencia en el habitus y lo performativo. Veámoslo.

Las escenificaciones se inspiran en el enfoque dinámico y situacionista desarrollado en la escuela antropológica británica de Manchester y su método de caso (Gluckman 1958; Mitchell 1956), proximidad propiciada por mi práctica con la escena naturalista. Como tales, aspiran a asemejarse a la definición de Clyde Mitchell sobre el análisis situacional, eel aislamiento intelectual de un conjunto de acontecimientos del contexto social más amplio en el que se inscriben con el fin de facilitar un análisis lógicamente coherente de esos acontecimientos" (en Mercier 1995: 163). Es más, los recortes situacionales de las escenificaciones pretenden adecuase al declarado por Gluckman como el uso más fructífero del método de los casos, que "consiste en considerar una serie de incidentes específicos, que afectan a las mismas personas o a los mismos grupos, durante un largo período de tiempo", para mostrar de qué modo estos casos "están unidos al desarrollo y al cambio de las relaciones sociales entre estas personas y estos grupos, actuando en el cuadro de su sistema social y cultural", y sugiere que los estudios más significativos son los que consideran «una serie de casos relacionados entre sí y que sobreviven en el mismo campo de vida social" (en Mercier 1995: 160-161). Las obras de Mitchell, "The Kalela Dance. Aspects of Social Relationships among Urban Africans in Northern Rodhesia" (1956), y de Gluckman, "Análisis de una situación social en Zululandia moderna. La organización social" (1958) reflejan así el estudio de un caso particular. En ellas elementos del orden social se expresan a través de los presentes en la situación. Del mismo modo, el boceto de escenificaciones presentado se inspira en el análisis situacional al abordar sus preocupaciones metodológicas acerca de la percepción de determinada situación social y así, mediante el análisis de los conflictos, contradicciones manifiestas o latentes en su seno, y que se desarrollan en el tiempo, extraer premisas generales sobre un delimitado aspecto de la vida social. Faltaría completarlo, claro está. En este caso, acotada su unidad de análisis, haciendo intervenir en ellas las interacciones del resto de productores/as y receptores/as de ese acontecimiento.

Además, la percepción completa de la unidad querría reproducir una estructura procesal que el alumno de Gluckman, Víctor Turner (1990, 1988 y 1987) define como "drama social", unidades de procesos sociales no armónicos que surgen en situaciones de conflicto. Porque en el boceto de escenificación propuesto, el conflicto o tensión entre naturaleza escénica y habitus, entre naturaleza y cultura o entre el híbrido naturaleza-escénica podría considerarse un estudio de caso sobre el proceso de resolución de ese conflicto-tensión, y seguir una estructura dramatúrgica con cuatro fases generales: quiebra, crisis, acción reconductora y reintegración/reconocimiento. Porque en la escenificación que gira en torno a escaleras y puertas del Sueño se percibe que 
contiene en cada etapa una fase liminal, la de la posibilidad, la de la hipótesis, las anticipaciones de la existencia posterior, entre la realidad y el sueño. En esta fase liminal, los participantes en el proceso ritual nos caracterizamos como indeterminados. Situados, betwixt and between ya no eramos lo que fuimos y aún no somos nada nuevo. Mas para Turner, el proceso ritual se instala en la tercera fase, la fase de acción reconductora a la que siguen los cambios sociales tras la crisis (1990, 1988 y 1987), una situación que en el Sueño se recondujo mediante la vuelta al palacio y nuestra participación en la boda de los personajes protagonistas, en un espacio situado betwixt and between el bosque de los sueños y la calle exterior al recinto. El acercamiento de Turner al paradigma teatral en sus análisis situacionales del proceso ritual y el drama social, inspirado por los rituales performativos del conflicto que defendía su maestro, puede considerarse pionero en el enfoque de la antropología de la performance como una nueva estrategia investigadora (Kolankiewicz 2008: 16). Fue mi primera inspiración y en consecuencia, tiendo a tomarlo como guía en gran parte de mis escenificaciones/percepciones etnográficas, principalmente en su tratamiento del conflicto/tensión como eje de la acción, del tiempo como transformación y cambio, y del espacio como geografía situada en fases liminares.

De igual forma, si del situacionismo he captado principalmente su estructura procesual y el recorte que establece en el conjunto de acontecimientos de cada caso, con Erving Goffman (2006, 1991, 1987 y 1979; Goffman y Díaz 2000) descubrí su enfoque dramatúrgico en el estudio de la interacción ${ }^{13}$ social. Por ello, la escenificación propuesta como ejemplo puede considerarse como un truncado marco interactivo que debiera progresar hacia la sutilidad, precisión y detalle de los microanálisis de este autor $^{14}$.

En Frame Analysis. Los marcos de la experiencia el autor declara no ocuparse de la estructura de la vida social, "sino de la estructura de la experiencia que los individuos tienen en cualquier momento de sus vidas sociales" (2006: 14). En este texto Goffman se ocupa de los marcos de referencia "para la comprensión y explicación del sentido de los acontecimientos" (2006: 10). Estos marcos, códigos o esquemas interpretativos, buscan una definición de la situación a partir de la pregunta: ¿qué es lo que está pasando aquí? En consecuencia, los marcos nos permiten encuadrar la experiencia, aquello con lo que una persona da sentido a una interacción, organizar su significado, pero también están implícitos en la conducta y guían la acción individual y colectiva. De tal forma que "la conducta ordinaria es, en cierto sentido, una imitación de los cánones sociales, un ademán dirigido a las formas ejemplares, y la realización primordial de estos ideales pertenece más al hacer creer, a la ficción, que a la realidad" (2006: 583). De este modo, las distintas escenificaciones abocetadas, para alcanzar su potencial, deberían beneficiarse del mismo tipo de enredo con el que Goffman describe la trasposición de claves en los marcos interactivos, entre otros encuadres y desencuadres de su obra. Con su misma atención al detalle, en los marcos de la interacción naturaleza-habitus.

\footnotetext{
13 George Hebert Mead instaura el principio central del interaccionismo simbólico con esta definición: "El yo, como aquello que puede ser un objeto para sí mismo, es esencialmente una estructura social y surge a través de la experiencia social" (en Hannerz 1986: 250).

${ }^{14}$ Obviamente, no hay espacio para ello en este artículo.
} 
Por otra parte, Goffman establece una analogía entre representar un papel en teatro y desempeñar un rol en la vida. Utiliza un modo de análisis basado en metáforas teatrales (actor, escenario, audiencia...), con el propósito de representar la interacción social como una sucesión de actuaciones y un proceso de gestión de las expresiones e impresiones. Su obra La presentación de la persona en la vida cotidiana, aparecida en 1959, investiga la naturaleza expresiva de las actividades humanas. En ella el autor detalla los modos en los que las personas presentan una imagen adecuada a sus intereses, que las hagan aceptables y creíbles. Esto es, en cada situación y cada interacción se requiere presentar una determinada imagen de sí mismas, y usar estratégicamente sus códigos expresivos, para tratar de manejar las impresiones y producir las reacciones que esperan de su audiencia. De esta forma, las interacciones estudiadas por Goffman pertenecen a la vida cotidiana e investigan cómo las personas manipulan la imagen que proyectan. Así que, siguiendo esta pauta, la escenificación propuesta se completaría con el análisis pormenorizado de los esfuerzos del público por mantener un habitus adecuado a pesar de la agencia de la naturaleza escénica. Y añadiría los marcos de los/as escenógrafos/as, políticos/as, productores/as y resto de agentes implicados.

Finalmente, un tercer desarrollo truncado en el bosquejo de escenificaciones propuesto gira en torno al tratamiento antropológico de los cuerpos presentes en esas situaciones e interacciones. Las teorías sociales del cuerpo han sido ampliamente incorporadas a las ciencias sociales desde que lo hiciera Marcel Mauss en 1934, como indica Mari Luz Esteban en su obra Antropología del cuerpo. Género, itinerarios corporales, identidad y cambio (2004). El campo de estudio del cuerpo ha incorporado también la filosofía, la semiótica, los estudios culturales, los estudios performativos y artísticos, y otros, de tal modo que al referirme en la unidad de análisis al habitus, he querido acotar entre todas posibilidades interpretativas, al enfocar los cuerpos sociales, la definida por Pierre Bourdieu de esta forma, "el habitus es a la vez un sistema de esquemas de producción de prácticas y un sistema de esfuerzos de percepción y de apreciación de las prácticas. Y, en los dos casos, sus operaciones expresan la posición social en la cual se ha construido" (1988: 107-108). En las siguientes escenificaciones que presento me valdré del habitus en relación al sistema de género, pero en estas se trata como una disposición corporal fuertemente ligada a la culturalización en campos sociales y sentidos estéticos condicionados por el gusto y en los que se revela su posición. Bourdieu desarrolló magistralmente este aspecto en La distinción. Criterio y bases sociales del gusto (2006) y en El baile de los solteros (2004). El modelo para mi bosquejo habría sido el capítulo 4 de este último texto, donde Bourdieu describe el habitus del campesino en el baile de Navidad, su choque con las técnicas corporales de las mujeres y hombres provenientes de la ciudad y su consecuencia sobre el celibato rural. Salvadas sean todas las distancias.

En cualquier caso, al valerme de la perspectiva de las escenificaciones durante el trabajo de campo he querido observar el comportamiento objetivamente perceptible de los grupos humanos, evaluar sus esquemas de acción y los diferentes mecanismos y lógicas de esa acción durante ciertas señaladas interacciones dramáticamente situadas. 


\section{LAS ESCENIFICACIONES COMO ESTRATEGIA ANALÍTICA}

Las escenificaciones como herramientas metodológicas pretenden ser un procedimiento susceptible de validarse $y$, en ese sentido, una estrategia analítica para evaluar empíricamente las hipótesis y así, ser instrumentos para la ciencia como "conocimiento que se pone sistemáticamente a prueba" (González 2006: 353). Esta validación, siguiendo a Paul Ricoeur sería «una disciplina argumentativa, comparable a los procedimientos jurídicos de interpretación legal" (2008: 72). Esto es, similar al razonamiento jurídico como proceso argumentativo donde el conflicto entre interpretaciones rivales se dirime en una interpretación final presentada en forma de veredicto que es posible apelar (Ricoeur 2008: 75).

Por tanto, las escenificaciones siguen un proceso de inducción a partir de los hechos observados particulares que intenta probar la hipótesis empíricamente mediante información detallada y fiable (González 2006: 332-333). En antropología, no obstante, explicamos los esquemas de acción de los individuos y los significados que ellos les otorgan. La ciencia antropológica, como conocimiento que se pone sistemáticamente a prueba, utiliza en su desarrollo procedimientos interpretativos (González 2006: 354). Buscamos correlaciones entre los fenómenos sociales, tratados como entidades semióticas (Ricoeur 2008: 79), dado que los actos humanos están esperando interpretaciones que decidan su significación, abiertos a una pluralidad de lecturas e interpretaciones (Ricoeur 2008: 68). Sin embargo, no todas las interpretaciones son equivalentes, siendo una interpretación más probable que otras (Ricoeur 2008: 73). ¿Cómo poner a prueba la coherencia de esa interpretación? Sin entrar en mayores detalles sobre el papel de la descripción en la obra Le savoir des antropologes de Sperber (1982), se validarían a través del contraste con otras interpretaciones (de la misma descripción) y la argumentación sobre su adecuación relativa ${ }^{15}$. En definitiva, mediante una articulación dialéctica entre la comprensión (hacer próximo lo lejano) y la explicación (extraer su estructura interna) (Ricoeur 2002: 182-195). Y finalmente, dado que "la cuestión de la ciencia en el feminismo trata de la objetividad como racionalidad posicionada" (Haraway 1995: 339), esta objetividad resultaría de una práctica de reflexividad sobre la propia subjetividad, de objetivar la propia posición y así permitir validar la interpretación.

Iniciaré ahora la ejemplarización de las escenificaciones como estrategia analítica, señalando que con la misma quiero corroborar si la naturaleza escénica es pertinente para el análisis de las categorías naturaleza, cultura y género. En primer lugar, utilizo para el análisis las clasificaciones de Philippe Descola en Mas allá de naturaleza y cultura (2012), pero como en esta obra las categorías sexo y género están ausentes o invisibilizadas, recurro luego a Marilyn Strathern y su texto No nature, no culture: the Hagen case (1980) para poner en cuestión la dicotomía occidental naturaleza/cultura e incidir sobre la construcción social del género (así como de la naturaleza escénica) y su capacidad de agencia. Marilyn Strathern discute que en todas las culturas se elabore la asociación del sexo con la naturaleza y el género con la cultura, ni de la mujer con la naturaleza y el hombre con la cultura (y las sucesivas dicotomías occidentales

\footnotetext{
${ }^{15}$ Agradezco enormemente a Aurora González Echeverría su pertinente revisión de una versión anterior de este apartado y su sugerencia sobre Paul Ricoeur (2002 y 2008).
} 
elaboradas sobre ella, como objeto/sujeto o subordinado/dominante). En la cultura Hagen, por ejemplo, la naturaleza no se manipula. Asimismo, destaca la interdependencia en las relaciones de género. Y en este marco, es igualmente congruente recurrir a Judith Butler (2007), quien entiende el género como una especie de transformación constante y repetida, activa y pasiva simultáneamente, en absoluto limitada por la dualidad aparente del sexo. En segundo lugar, la construcción de las escenificaciones propuestas sigue pautas teóricas complementarias a las expuestas en el anterior apartado, que serán detalladas a posteriori. Evidentemente, mostraré sólo unos fragmentos del análisis. Este sigue así.

Descola presenta cuatro esquemas clasificatorios implícitos en las diversas maneras de organizar la interacción naturaleza y cultura; principios de identificación que definen cuatro grandes tipos de ontología: animismo, totemismo, naturalismo y analogismo. Estos esquemas de integración de las experiencias, afirma Descola (2012: 345), coexisten en potencia en cada ser humano, aunque se movilicen y lleguen a ser dominantes en ciertas situaciones históricas. Las obras de "naturaleza escénica", sospecho, tienen capacidad de movilizar esquemas no dominantes, además del dominante en nuestro contexto (naturalismo). Txoriei activaría el analogismo, una ontología que establece una densa red de analogías entre múltiples esencias, formas y sustancias. El espectáculo, que según el director de la orquesta fusiona ornitología y música, activaba correlaciones entre las réplicas de las flautas de hueso encontradas en las cuevas de Uztariz, que tocaba Mixel Etxekopar, y el piano de cola cuyas cuerdas rasgaba François Rossé y, asimismo, suspendía las diferencias entre las composiciones modernas influenciadas por Olivier Messiaen (sus cantos de pájaros) y la música popular vasca, o establecía resonancias entre el canto, en sí mismo fusión entre naturaleza y cultura, y los recitados poéticos. La construcción emocional Txoriei implicaba a la personalidad en un nivel profundo donde el juego de conexiones desdibujaba las fronteras dicotómicas. Podría seguir. Con el efecto totémico de la escultura Besarkada (abrazo) de Chillida sobre los cinco bailarines que a su alrededor dibujaban figuras del mandala con sus brazos, mientras se ejecutaba en un piano de cola una delicada melodía. O las lecturas animistas de las escenificaciones con personajes del folklore vasco joalduna (caminantes con cencerros, vestidos con piel de oveja, hisopo de cola de caballo, gorro cónico, combinación de puntillas, pantalón de mahón y albarcas) y zaldiki/zamalzaina (danzantes mitad persona, mitad caballo) en Topaguneak, o con las hadas y duendes, la flor hechicera y el artesano con cabeza de burro del Sueño. Pero es más pertinente seguir con la ilustración del cuestionamiento de la dicotomía naturaleza/cultura en la categoría género, que paso a escenificar en las dos siguientes secuencias.

La coreografía elegida de Topaguneak se elaboró por cinco danzantes: completamente de blanco, medias caladas, falda corta de vuelo, pantalón de media pierna con adornos, cara cubierta con una matilla de encaje. Un traje que facilita la confusión de géneros. Crearon una figura suplicante, con las manos entrelazadas en gesto de rezo, y la torsión de brazos, tronco y piernas construía una presencia femenina. Los movimientos suaves, ligeros o recogidos alternaban con otros enérgicos y varoniles. Se quitaron el velo, bailaron; luego la falda, bailaron. La gestualidad creció en vigor. La coreografía fusionaba danza contemporánea y tradicional vasca y dentro de esta última, alternaba pasos de baile codificados para hombres y para mujeres. Los bailarines 
—en sus saltos, giros, corros- modelaban su energía con delicadeza o con vigor, la tensión de fuerzas opuestas se resolvía en movimientos refinados o violentos. En definitiva, esculpieron con sus cuerpos la ficción del sexo, del género.

La puesta en escena del Sueño se desarrollaba en tiempos actuales. El director decidió también que Lisandro fuera Lisandra y así, que el amor prohibido de Lisandro por Hermia desafiara la heteronormatividad. El enredo amoroso ganaba en inteligibilidad, a nuestros ojos. Shakespeare escribió muchos personajes que disfrazados del sexo opuesto intervenían en enredos amorosos. En su época, además, las mujeres eran representadas por chicos jóvenes. Pero en el Sueño una mujer actuaba como tal en un papel escrito para un hombre. La actriz, sin poner en cuestión su sexo, intervenía en una secuencia de acciones imaginada para un hombre enamorado. Ella proponía la huida al bosque, ella conducía la moto donde escaparon, ella actuó pícara y caballerosamente al caer la noche, despertó enamorada de Helena, aborreció a Hermia y peleó con Demetrio por su nuevo amor. Cualquiera de sus acciones había sido escrita para un hombre; y analizadas en correlación con la dicotomía naturaleza/cultura les correspondía el segundo elemento de los consecuentes pares, paciente/agente, objeto/sujeto, subordinado/dominante, etc. La mujer Lisandra encarnaba todos ellos -agente, sujeto, dominante-, subvirtiendo así la creencia de que el comportamiento está biológicamente programado y violando el orden de lo considerado natural en la tradición europea. Esto es, mostrando cómo el cuerpo es una ficción social y la posibilidad de reestructurar las relaciones asimétricas entre los sexos.

A continuación incidiré en lo que hace estas escenificaciones potencialmente válidas para la investigación antropológica feminista.

Coincido con Linda Alcoff en que «la subjetividad femenina está construida aquí y ahora de esta forma" (1989: 14), sin que ello implique una máxima universalizable sobre lo femenino sino un concepto posicional que incluye dos puntos: el primero señala que el concepto de mujer es un término relacional, identificable sólo dentro de un contexto histórico (en constante movimiento), y su posición dentro de esa red de relaciones constituiría su identidad; y el segundo punto afirma que esa posición puede ser activamente utilizada, puede reconstruirse en cada momento histórico y alterar ese contexto (Alcoff 1989). Siguiendo este concepto posicional y relacional, las escenificaciones presentadas, aún arrastrando las mismas carencias que las del anterior apartado, actúan como artefactos para desarrollar una visión constructivista del género. Y en consecuencia, recurren a teorías que trabajaran esta construcción desde lo performativo.

En primer lugar, rescato la argumentación de Elin Diamond sobre la potencialidad de la técnica de extrañamiento de Brecht para el feminismo (Diamond 1997: 48-49). Esta técnica, también llamada efecto $V^{16}$, crea distancia y análisis respecto a los acontecimientos al establecer una ruptura en el curso de la historia que permite intervenir y posicionarse en la acción ${ }^{17}$ (en Ceballos 1992). Dicho de otro modo, el efecto $V$ sir-

\footnotetext{
${ }^{16}$ Efecto $V$, en alemán Verfremdungseffect, que significa 'efecto de extrañamiento', 'efecto de distanciamiento'.

${ }^{17}$ En su artículo "Teatro épico", publicado en Principios de dirección escénica de Edgar Ceballos, dice Bertolt Brecht: "Esta técnica del verfremdungseffekt o efecto de distanciamiento tiene por objeto colocar al espectador en una actitud inquisidora, crítica, frente al proceso representado" (...) "...es decir, no se pretenderá hipnotizar al público ni crearle la ilusión de que se halla en presencia
} 
ve para hacer entender las relaciones de género como parte de una dialéctica en proceso y, de este modo, descubrir las limitaciones de la propia realidad para poder comenzar a cambiarla, pues tras la acción se vislumbran las posibilidades de lo que no está siendo representado (Diamond 1997). Por tanto, el efecto $V$ desnaturaliza lo que la ideología y la performatividad hacen aparecer normal y así, sirve al propósito de desnaturalizar la ideología de género, sistema de creencias cuya performatividad hace parecer normal, aceptable, natural, esa ideología (Diamond 1997). Y ese distanciamiento es el que me ha permitido analizar las escenificaciones propuestas, más allá de la naturalización del género.

En segundo lugar, para explicitar el modo de análisis en el boceto de las escenificaciones de Topagune y Sueño debo descubrir la importancia que algunos textos y experiencias han tenido en mi acercamiento al concepto de género/sexo social, como es el caso de El arte secreto del actor. Diccionario de Antropología Teatral, de Eugenio Barba y Nicola Savarese. Por ejemplo, allí estudié que el antropólogo Marcel Mauss había hablado por primera vez en 1934 de las "técnicas del cuerpo", es decir, de las formas en que las personas, en las distintas sociedades, utilizan, de acuerdo con la tradición, su propio cuerpo (Barba y Savarese 1990: 300-305). Este rechazo implícito de la idea de cuerpo natural, dado que en él dominan los modelos sociales sobre los biológicos, me aproximó a la noción del cuerpo como ficción social, y esta a la concepción del género como proyección de imágenes codificadas y encarnadas como masculinas o femeninas. Perfilé estos conceptos con Mari Luz Esteban y su texto $A n$ tropología del cuerpo de (2004). Y ese texto de Barba y Savarese (1990) fue también el que me hizo reflexionar ${ }^{18}$ sobre cómo la androginia, arquetipo de la reunión de lo masculino y lo femenino, ha sido escenificada en el teatro kabuki, el teatro renacentista ingles y el teatro del siglo de Oro español ${ }^{19}$, de manera que puede hablarse de esos actores y actrices que representaban al otro sexo como un "tercer sexo/género", un cuerpo andrógino que transgrede simbólicamente la creencia de que el comportamiento está biológicamente y sexualmente programado. Texto del que claramente son deudores ambos bocetos de escenificaciones presentados.

En tercer y último lugar, para el análisis performativo de la identidad ${ }^{20}$ y las relaciones de género en las escenificaciones debo volver a citar como mínimo a Bourdieu

de un suceso natural no ensayado" (...) "La condición indispensable para que se produzca el efecto de distanciamiento consiste en que el actor emplee un claro gesto demostrativo para señalar lo que tiene que manifestar. Lógicamente, debe abandonarse la idea de que existe una cuarta pared imaginaria que separa al escenario del público, creando la ilusión de que el proceso escénico se está desarrollando en la realidad, sin la presencia del público. Es fundamental que el actor encare directamente al público" (en Ceballos 1992: 253).

${ }^{18}$ Escribí un trabajo durante los cursos de doctorado con Mari Luz Esteban y Carmen Diez Mintegi, que ha sido publicado recientemente con el título "Reconfiguración del género en las performances clásicas. Androginia actoral” (Urquijo 2015).

19 Tomé los casos en los que actores y actrices representaban personajes del otro sexo en el teatro tradicional japonés kabuki, en el teatro renacentista ingles y el teatro del siglo de Oro español.

${ }^{20}$ Sobre identidad plural Bernard Lahire (2005) sostiene que el actor es plural, al ser producto de socializaciones heterogéneas y estar llamado a tener comportamientos y actitudes variadas, según los contextos en los que se desenvuelve. Para Bruno Latour "a los actores humanos completos hay que componerlos a partir de muchas capas sucesivas, cada una empíricamente distinta de la siguiente" (2008: 276). Ambas propuestas pueden ser interpretadas desde una perspectiva feminista. 
y Butler ${ }^{21}$. Pueden establecerse equivalencias entre ellos. Para Butler el género es performativo y "la reproducción de género es siempre una negociación de poder" (2009: 322). Así, el género posee una determinada expresión, condicionada por normas obligatorias que actúan sobre nosotros antes de que tengamos la ocasión de actuar, y se abren camino en nuestra propia acción (Butler 2009: 333). El poder, para mantenerse, necesita reproducirse, pero cada acto de reproducción puede resultar desafortunado y producir efectos no pronosticados (Butler 2009: 323): "Yo estoy, en mi deseo, negociando lo que se ha querido de mí" (2009: 333).

Desde esta perspectiva entendí a Pierre Bourdieu, su concepto de habitus y su teoría sobre la dominación masculina (2000), porque el habitus, en cuanto sistema de esquemas de clasificación, implica que el mundo social se presente como una realidad fuertemente estructurada (1988: 109). En La dominación masculina (2000) se analiza la estructura de dominación que permite las relaciones asimétricas entre los sexos. Para Bourdieu los dominados aplican los esquemas de percepción de los dominantes, pues la estructura de dominación se inscribe en los cuerpos, a partir de la naturalización corporal de las estructuras dicotómicas masculino/femenino, e impone con el habitus su visión de las clasificaciones. Las personas dominadas, quienes aceptan los límites impuestos por el sistema de clasificaciones dominante, son las destinatarias de la violencia simbólica generada en las luchas por el poder simbólico y performativo de designación, de imponer el propio habitus (1988: 112-113). La estructura de dominación subyace a pesar de los procesos de cambio, pero aún así existe una pluralidad de estructuraciones posibles y sería posible neutralizarla.

Estas equivalencias entre Bourdieu y Butler en su tratamiento sobre la performatividad del género, su apreciación sobre el papel de la normatividad y la estructura social en su representación y asimismo, volviendo a Alcoff (1989), en su reconocimiento de la posición que puede reconstruirse y alterar el contexto histórico, sostienen el análisis de las escenificaciones del baile andrógino de Topaguneak o el juego de roles en el Sueño. Pero más allá de este análisis, todas las escenificaciones descritas, con el resto del las no formuladas, se encadenarían y estructurarían para así poder evaluar empíricamente mi hipótesis de investigación, esto es, que la naturaleza escénica es "buena para pensar" las relaciones de continuidad y discontinuidad entre naturaleza, cultura y género. Haciendo de ella un veredicto que es posible apelar.

\section{CONCLUSIONES}

En las escenificaciones intervienen elementos constitutivos del drama. Es decir, las/ los actores, sus relaciones y sus situaciones conflictivas. Son también herramientas de la antropología feminista. Así, al focalizar sobre las/los actores, se centran en comprender y explicar sus agencias, vivencias, experiencias y expresiones de sus cuerpos como ficciones sociales. Asimismo, persiguen interpretar sus relaciones/interacciones/ intersubjetividades desde su identidad posicional, su pluralidad de lógicas de acción

\footnotetext{
${ }^{21}$ Para el análisis de las relaciones de género resulta también aplicable la propuesta de Goffman, quien en "La ritualización de la feminidad" (1991) hizo un estudio de las escenificaciones que las mujeres deben seguir deliberadamente para los fotógrafos publicitarios.
} 
y la adecuación normativa a sus intereses. Y finalmente, pretenden explicar sus marcos situacionales, posicionales, conflictivos, dinámicos e históricos, desde esquemas de clasificación, para la percepción y la producción/expresión de experiencias, y desde las estructuras de dominación inscritas en sus cuerpos, sus experiencias (y expresiones) condicionadas por el poder normativo del sistema de género.

\section{BIBLIOGRAFÍA CITADA}

Abrahams, Roger D. 1986. "Ordinary and Extraordinary Experience", en Victor Turner y Edward Bruner (eds). The Anthropology of experience: 45-72. Illinois: University of Illinois Press.

Alcoff, Linda. 1989. "Feminismo cultural versus pos-estructuralismo: la crisis de la identidad en la teoría feminista". Revista Feminaria 4: 1-18.

Barba, Eugenio y Nicola Savarese. 1990. El Arte Secreto del Actor. México: Escenología.

Bourdieu, Pierre. 1988. "Espacio social y poder simbólico". Revista de Occidente 81: 97-119.

Bourdieu, Pierre. 2000. La dominación masculina. Barcelona: Anagrama.

Bourdieu, Pierre. 2004. El baile de los solteros. Barcelona: Anagrama.

Bourdieu, Pierre. 2006. La distinción. Criterio y bases sociales del gusto. Madrid: Taurus.

Brecht, Bertolt. 1983. El pequeño organón para el teatro escrito en 1948. Granada: Don Quijote.

Butler, Judith. 2007. El género en disputa. El feminismo y la subversión de la identidad. Barcelona: Paidós.

Butler, Judith. 2009. "Performatividad, precariedad y políticas sexuales". AIBR Revista de Antropología Iberoamericana 4/3: 321-336.

Ceballos, Edgar. 1992. Principios de dirección escénica. México: Escenología.

Descola, Philippe. 2012. Más allá de naturaleza y cultura. Buenos Aires: Amorrortu.

Diamond, Elin. 1997. Unmaking Mimesis. Nueva York: Routledge.

Esteban, Mari Luz. 2004. Antropología del cuerpo. Género, itinerarios corporales, identidad y cambio. Barcelona: Bellaterra.

Firscher-Lichte, Erika. 2011. Estética de lo performativo. Madrid: Abada.

Gell, Alfred. 2016. Arte y agencia. Una teoría antropológica. Buenos Aires: SB.

Gluckman, Max. 1958. "Análisis de una situación social en Zululandia moderna. La organización social". Rhodes Livingstone Paper 28: 1-27. Disponible en: <http://www.ciesas.edu.mx/publicaciones/Clasicos/00_CCA/Articulos_CCA/CCA_PDF/031_GLUCKMAN_Analisis_de_una_situacion.pdf> Fecha de acceso: 04 jun. 2016.

Goffman, Erving. 1979. Relaciones en público. Microestudios de orden público. Madrid: Alianza.

Goffman, Erving. 1987. La presentación de la persona en la vida cotidiana. Buenos Aires: Amorrortu.

Goffman, Erving. 1991. "La ritualización de la feminidad", en Erving Goffman e Yves Winkin (comp.), Los momentos y sus hombres: 135-168. Barcelona: Paidós.

Goffman, Erving. 2006. Frame Analysis. Los marcos de la experiencia. Madrid: CIS/Siglo XXI.

Goffman, Erving y Félix Díaz. 2000. Sociologías de la situación. Madrid: La Piqueta.

González Echeverría, Aurora. 2006. "Del utillaje conceptual de la antropología: los usos del término "inductivismo" y los usos del término "hermeneútica". Dos propuestas de clarificación". Revista de Antropología Social 15: 327-372.

Haraway, Donna J. 1995. Ciencia, cyborgs y mujeres. La reinvención de la naturaleza. Madrid: Cátedra.

Hannertz, Ulf. 1986. Exploración de la ciudad. México: Fondo de Cultura Económica.

Hernández, Jone Miren. 1999. "Auto/biografía, Auto/etnografía, Auto/retrato". Ankulegi 3: 53-62.

Kolankiewics, Leszek. 2008. "Towards an Anthropology of Performance(s)". Performance Research: A Journal of the Performing Arts 13(2): 8-24. doi: 10.1080/13528160802639177

Lahire, Bernard. 2005. El hombre plural. Los resortes de la acción. Barcelona: Bellaterra.

Latour, Bruno. 2008. Reensamblar lo social. Una teoría del actor-red. Buenos Aires: Manantial.

Mercier, Paul. 1995. Historia de la antropología. Barcelona: Península. 
Mitchell, Clyde. 1956. The Kalela Dance. Aspects of Social Relationships among Urban Africans in Northern Rodhesia. Manchester: Manchester University Press.

Ricoeur, Paul. 2002. Del texto a la acción. Ensayos de Hermeneútica II. México: Fondo de Cultura Económica.

Ricoeur, Paul. 2008. Hermeneútica y Acción. De la Hermenútica del Texto a la Hermeneútica de la Acción. Buenos Aires: Prometeo.

Sperber, Dan. 1982. Le savoir des anthropologues: Trois essais. París: Hermann.

Strathern, Marilyn. 1980. "No nature, no culture: the Hagen case", en Carol MacCormack y Marilyn Strathern, Nature, Culture and Gender: 174-222. Cambridge: Cambridge University Press.

Turner, Victor. 1987. The Antbropology of Performance. Nueva York: PAJ Publications.

Turner, Victor. 1988. El proceso ritual: estructura y antiestructura. Madrid: Taurus.

Turner, Victor. 1990. La selva de los símbolos: aspectos del ritual nbembu. Madrid: Siglo XXI.

Turner, Victor y Edward M. Bruner. 1986. The Anthropology of experience. Illinois: University of Illinois Press.

Urquijo, Miren. 2011. "Construir distancia como estrategia metodológica. El argumento de las escenificaciones". Ankulegi 15: 81-89.

Urquijo, Miren. 2015. "Reconfiguración del género en las performances clásicas. Androginia actoral". Perifèria 20(2): 72-89.

Urquijo, Miren. 2016. "Eszenaratzeak, antropologiaren tresna metodologiko", en Mari Luz Esteban y Jone M. Hernández (coord.). Etnografia feministak Euskal Herrian. XXI. Mendera begira dagoen antropología: 193-207. Bilbao: Udako Euskal Unibertsitatea-Euskal Herriko Unibertsitatea.

Valle, Teresa del. 1995. "Metodología para la elaboración de la autobiografía", en Actas del Seminario Internacional "Género y trayectoria del profesorado universitario": 281-289. Madrid: Instituto de Investigaciones Feministas/Universidad Complutense.

Fecha de recepción: 5 de enero de 2017

Fecha de aceptación: 8 de junio de 2017 